\title{
FIBROELASTOMA PAPILÍFERO DE CORDOALHA MITRAL EM PACIENTE OCTAGENÁRIO: ESTUDO DE CASO
}

\author{
PAPILLARY FIBROALASTOMA OF MITRAL CHORDAEA IN \\ OCTAGENOUS PATIENT: CASE STUDY
}

\author{
Jaqueline Trentin ${ }^{1 *}$, Ana Paula Susin Osório², Kleiton Marques de Oliveira³ \\ ${ }^{1}$ Acadêmica do Curso de Medicina do Centro Universitário Assis Gurgacz - FAG ${ }^{2}$ Cardiologista do \\ Corpo Clínico do Hospital São Lucas - HSL ${ }^{3}$ Cardiologista do Corpo Clínico do Hospital São Lucas - \\ HSL, Professor do Curso de Medicina do Centro Universitário Assis Gurgacz - FAG Preceptor da \\ Residência Médica em Clínica Médica do Hospital São Lucas
}

*Autor correspondente: jatrentin@gmail.com https://orcid.org/0000-0003-4157-5807

\section{RESUMO}

Introdução: os tumores cardíacos são neoplasias raras, frequentemente assintomáticos e diagnosticados incidentalmente. São classificados como primários e secundários. Suas principais complicações são a embolização sistêmica e o acidente vascular cerebral. Por possuírem clínica inespecífica, é importante um diagnóstico apurado, baseado em exames complementares, a fim de evitar suas possíveis complicações e traçar um plano terapêutico com bom prognóstico. Relato do caso: paciente do sexo masculino procurou atendimento cardiológico ambulatorial por Hipertensão Arterial Sistêmica não controlada mesmo com uso correto das medicações. Submetido a um ecocardiograma transtorácico, que evidenciou uma massa compatível com fibroelastoma papilífero (FEP). Paciente continua em acompanhamento ambulatorial cerca de um ano após o diagnóstico, assintomático e sem complicações. Discussão: o FEP é um tumor que acomete principalmente as válvulas cardíacas, por volta dos 50 anos. Possui clínica inespecífica e seu tratamento definitivo é cirúrgico. O caso relata um paciente octagenário assintomático, diagnosticado com FEP em cordoalha e com tratamento ambulatorial. Conclusão: 0 FEP é uma condição rara e de difícil diagnóstico. Embora benigno, suas complicações podem ser fatais. Nesse contexto, é importante pesquisar eventos prévios de origem coronariana ou cerebrovasculares e fazer o uso de exames complementares para direcionar ao diagnóstico correto, evitando possíveis complicações.

Palavras-chave: Tumores cardíacos. Cordoalha mitral. Fibroelastoma papilífero.

\begin{abstract}
Introduction: cardiac tumors are rare neoplasms, often asymptomatic and incidentally diagnosed. They are classified as primary and secondary tumors. The main complications are systemic embolization and stroke. Due to the fact that they have a nonspecific clinical, is important an accurate diagnosis, based on complementary tests is in order to avoid their possible complications and to draw up a therapeutic plan with good prognosis. Case report: male patient sought out outpatient cardiac care due to uncontrolled systemic arterial hypertension even with correct use medications. Undergoing a transthoracic echocardiogram to control, the examination revealed a mass compatible with papillary fibroelastoma (PFE). Patient continues in outpatient follow-up about one year after diagnosis, asymptomatic and free complications. Discussion: PFE is a tumor that mainly affects the heart valves, around the age of 50
\end{abstract}


years. The definitive treatment is surgical. The case reports an asymptomatic octagenous patient, diagnosed with PFE in the mitral chordaea and with outpatient. Conclusion: PFE is a rare condition and difficult to diagnose. Although benign, the complications can be fatal. In this context, it is important to investigate previous events of coronary or cerebrovascular origin and to make use of complementary exams to direct the correct diagnosis, avoiding possible complications.

Keywords: Cardiac tumors. Mitral chordaea. Papillary fibroalastoma.

\section{INTRODUÇÃO}

Os tumores cardíacos são neoplasias raras que podem se originar de qualquer tecido cardíaco ou serem manifestações metastáticas de outros órgãos. Podem ser classificados como primários ou secundários, a depender de seu poder metastático ou não. A maioria dos tumores cardíacos (75\%) são benignos e o restante $(25 \%)$ apresenta algum grau de malignidade. (KARIGYO; SILVA, 2014) A incidência dos tumores primários é menor que $0,1 \%$ enquanto que a dos tumores secundários é cerca de 20 a 40 vezes maior. (CORSO, 2003) Até recentemente, o diagnóstico dessas afecções cardíacas era eminentemente cirúrgico ou feito através de necrópsia. Nas últimas décadas, com a inserção de novos métodos complementares na área da cardiologia, principalmente do ecocardiograma, houve um aumento na taxa de diagnósticos, permitindo uma abordagem precoce e prevenindo complicações. (REYKEN, 1996)

Em sua grande maioria, os pacientes são assintomáticos. Porém, dependendo da localização, tamanho e expansividade do tumor, podem apresentar sintomas. Quando presentes, são inespecíficos e podem mimetizar outras patologias cardíacas ou até sistêmicas, sendo necessária uma análise mais apurada com métodos complementares para o diagnóstico. (GOUVEIA, 2010)

As complicações mais temidas diante de um tumor cardíaco, mesmo estas sendo raras, são a embolização sistêmica e o acidente vascular cerebral (AVC), podendo ser fatais. (GOWDA, 2003)

Os tumores primários são em sua maioria benignos e representados pelos lipomas, rabdomiomas, fribromas e os fibroelastomas papilares. Em média, 50\% desses tumores é representado pelo mixoma na fase adulta. (KARIGYO; SILVA, 2014)

Tanto para Karigyo e Silva (2014) como para Oliveira et al (2005), o fibroelastoma papilífero (FEP) é um tumor primário e benigno que corresponde a $8 \%$ dos tumores cardíacos, sendo o terceiro tumor cardíaco mais comum. 
Relatamos a seguir um caso clínico sugerido de FEP, com diagnóstico ao acaso em paciente assintomático. Destaca-se a importância dos exames complementares, sobretudo do ecocardiograma, no delineamento do diagnóstico até a confirmação do mesmo.

\section{METODOLOGIA}

A pesquisa desse estudo de caso constitui-se de forma qualitativa e descritiva. O artigo foi elaborado por meio do prontuário médico e exames de imagem desse paciente, com o consentimento do mesmo, obtido através do termo de assentimento. A pesquisa foi realizada no Hospital São Lucas de Cascavel. O projeto de pesquisa foi ao Comitê de Ética e Pesquisa do Centro Universitário FAG.

\section{RELATO DO CASO}

Paciente do sexo masculino, 85 anos, aposentado, procurou atendimento cardiológico ambulatorial por Hipertensão Arterial Sistêmica (HAS) não controlada. Encontra-se assintomático do ponto de vista cardiovascular. Negava histórico prévio de eventos cardiovasculares, como infarto agudo do miocárdio (IAM) ou AVC.

Figura 1: Corte apical 3 câmaras evidenciando massa arredondada, bem delimitada, aderida à cordoalha relacionada ao folheto anterior da válvula mitral, compatível com fibroelastoma papilífero.

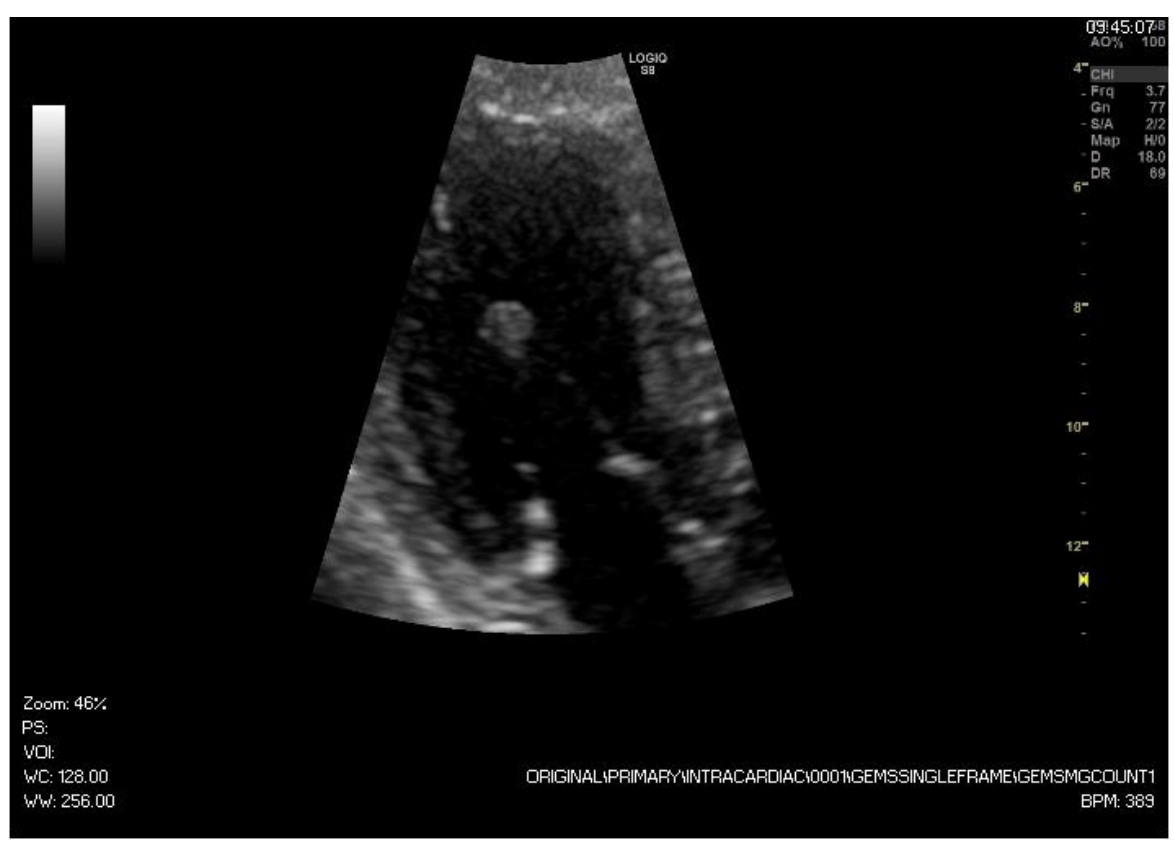

Ao exame físico, encontrava-se em bom estado geral, lúcido e orientado, acianótico, anictérico e afebril. A pressão arterial aferida foi de 210x120 mmHg. 
Ausculta cardíaca com ritmo regular, 2 tempos, bulhas normofonéticas e ausência de sopros. Não havia outras particularidades ao exame. O eletrocardiograma de repouso mostrava ritmo sinusal e não havia alterações dignas de nota.

Figura 2: Corte apical 3 câmaras mostrando fibroelastoma papilífero medindo cerca de 0,88 x 0,85 cm.

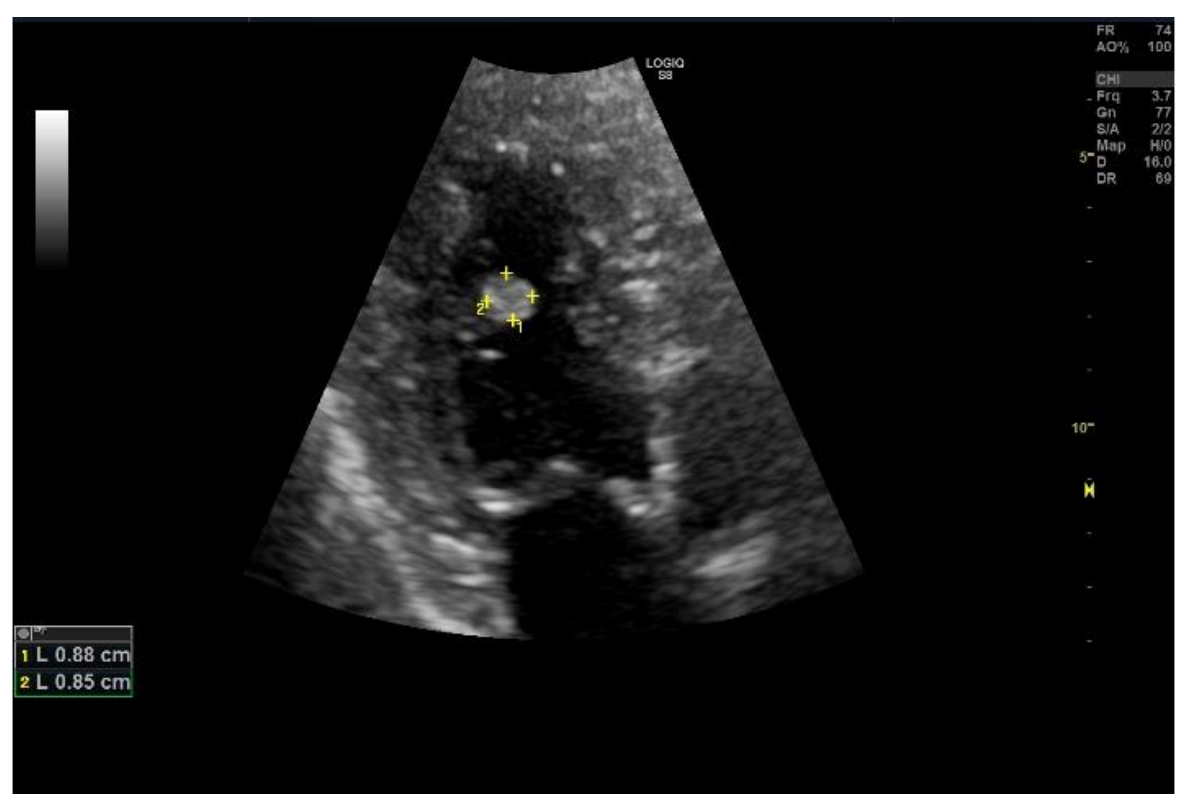

Durante a avaliação, foi realizado o ajuste das medicações anti-hipertensivas e solicitados exames laboratoriais e ecocardiograma transtorácico (ETT) devido HAS não controlada de longa data.

Figura 3: Estruturas cardíacas visualizadas na janela apical eixo longo.

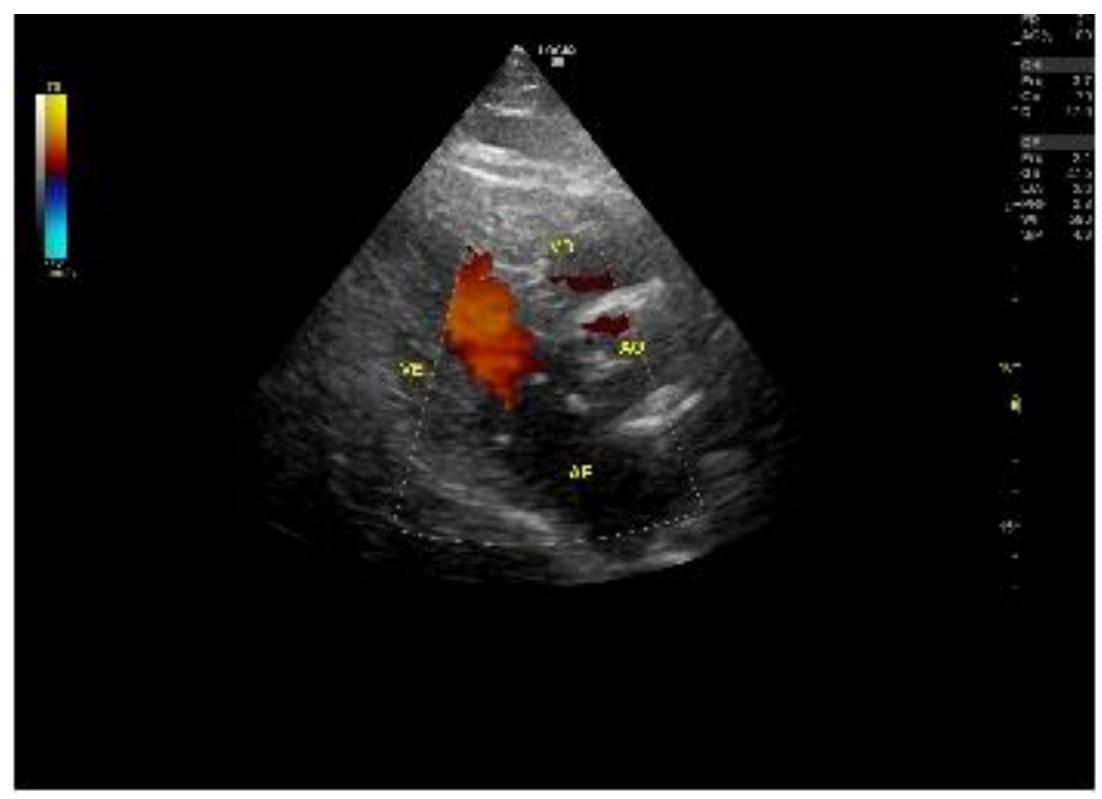

Ao ETT, foi visualizada uma massa arredondada, pediculada, bem delimitada, móvel, medindo $0,88 \times 0,85 \mathrm{~cm}$, aderida à cordoalha relacionada ao folheto anterior 
da válvula mitral - achados compatíveis com FEP (figuras 1 e 2). Além disso, o exame mostrava sobrecarga de átrio esquerdo, regurgitação mitral de grau mínimo, esclerose valvar aórtica com regurgitação mínima (figura 3) e ventrículo esquerdo com dimensões preservadas, hipertrofia de grau leve, disfunção diastólica grau I e função sistólica normal (fração de ejeção de 68,77 \%).

Figura 4: Estruturas cardíacas visualizadas na janela apical 4 câmaras.

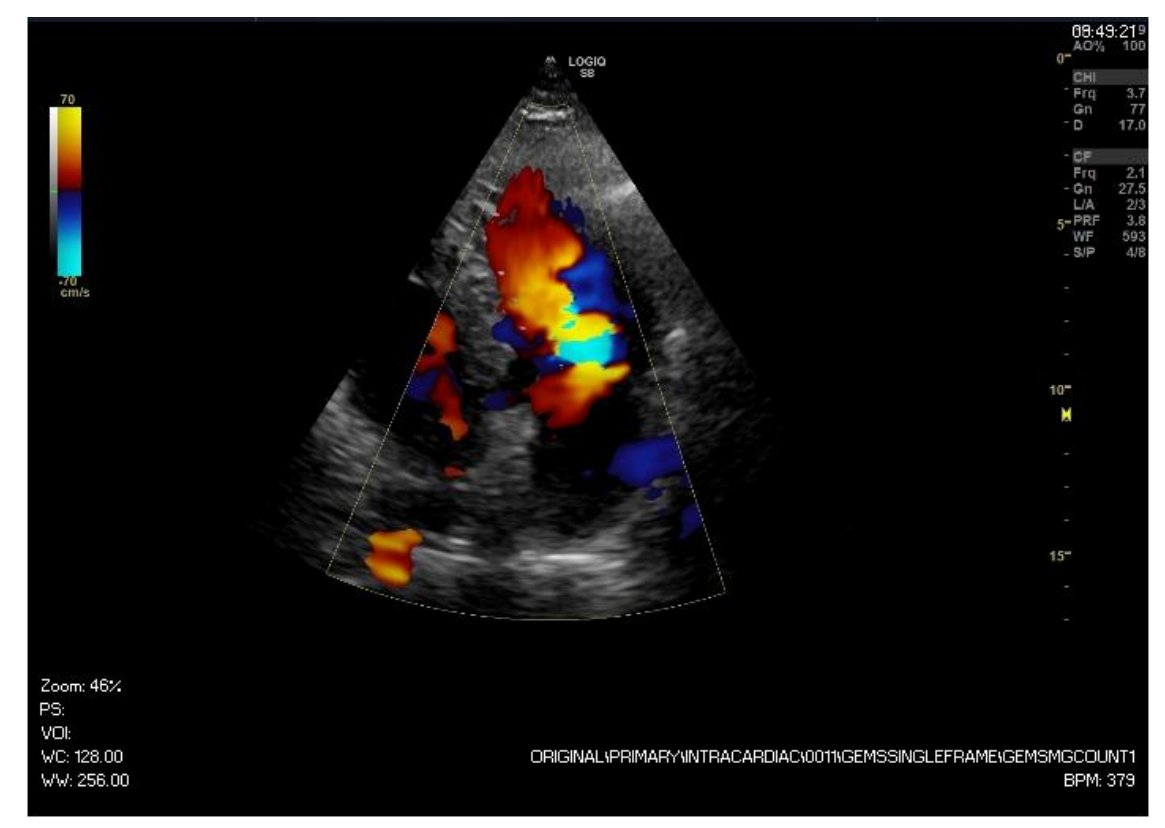

O paciente foi reavaliado após a realização do exame, e por decisão conjunta da equipe de Cardiologia, tendo em vista a idade do paciente e ausência de fenômenos embólicos e/ou sintomas em sua história, optou-se por conduta conservadora. No seguimento, o paciente continua em acompanhamento ambulatorial, assintomático e livre de eventos cerca de um ano após o diagnóstico.

\section{DISCUSSÃO}

O FEP é um tumor benigno do coração que corresponde a aproximadamente $8 \%$ dos tumores cardíacos. É um tumor primário raro que acomete principalmente as válvulas cardíacas. (OLIVEIRA et al., 2005) É uma neoplasia derivada do endocárdio e aderida a este por um pedículo curto com prolongamentos papilares avasculares que se assemelham a forma de anêmonas do mar, sendo assim comparado. (KARIGYO; SILVA, 2014) São encontrados na maioria das vezes incidentalmente e frequentemente confundidos com vegetações. (ABAD, 1998) 


\section{FAG JOURNAL OF HEALTH}

É o terceiro tumor cardíaco benigno mais comum na fase adulta, ficando atrás somente do mixoma e do lipoma. (GOUVEIA, 2010) A idade de apresentação mais comum é a partir dos 50 anos, não havendo diferenciação entre os sexos. (OLIVEIRA et al, 2005) Quanto ao seu tamanho, a maioria desses tumores são pequenos e seu maior diâmetro mede menos de $2 \mathrm{~cm}$. (GOUVEIA, 2010)

As válvulas cardíacas são acometidas em cerca de $90 \%$ dos FEP, sendo esse acometimento com lesões únicas e raramente múltiplas. (KARIGYO; SILVA, 2014) Nas válvulas aórticas são encontrados cerca de $44 \%$ dos FEP, seguido de $35 \%$ do acometimento em válvula mitral, $15 \%$ das válvulas tricúspides e $8 \%$ nas pulmonares. (OLIVEIRA et al., 2005) FEP aderidos em cordoalhas são ainda mais raros e com poucos relatos na literatura.

O caso relatado demonstra perfil epidemiológico e apresentação semelhante ao da literatura, mas possui discrepância em relação a localização, sendo este relatado um FEP de cordoalha mitral.

Gouveia (2010) e Oliveira et al (2005) elucidam que, assim como os outros tumores cardíacos, o FEP pode ser assintomático ou ter manifestações clínicas dependendo do tamanho, mobilidade e localização do mesmo. Cerca de $95 \%$ dos FEP originam-se das câmaras esquerdas, tendo assim, como complicação mais temida a embolização sistêmica, principalmente para áreas cerebrais causando um AVC ou para a região coronariana, causando IAM. Gouveia (2010) juntamente com Lima e Crotti (2004), relatam além dessas, outras manifestações como angina, insuficiência cardíaca, síncope, embolia pulmonar, arritmias e até mesmo morte súbita podem ocorrer.

No caso demonstrado, o paciente apresentava-se de forma assintomática e sem história prévia de AVC ou IAM.

O ecocardiograma transesofágico é o melhor exame complementar para diagnóstico, com sensibilidade de $88,9 \%$ e especificidade de $87,8 \%$ para tumores maiores que $20 \mathrm{~mm}$. Para dimensões menores, a sensibilidade do exame fica em 61,9\%. MARISCALCO et al., 2010) O exame caracteriza o tumor quanto tamanho, mobilidade, delimitações e formato. (GOUVEIA, 2010) As características do FEP ao ecocardiograma mostram-se como hiperecogênicas, homogêneas, bem delimitadas, pedunculadas ou sésseis, de formato arredondado, oval ou irregular. (OLIVEIRA et al., 2005) 


\section{FAG JOURNAL OF HEALTH}

Oliveira et al (2005) aponta outros exames que podem auxiliar no diagnóstico, como o eletrocardiograma, com achados inespecíficos, desde arritmias até bloqueios de ramos. A radiografia de tórax, que dependendo da localização do tumor, pode haver sinais que sugerem hipertensão e congestão pulmonar, bem como aumento de câmaras cardíacas e a ressonância nuclear magnética com o uso de gadolínio para realce tumoral que aumenta a suspeita de tumor. (OLIVEIRA et al., 2005)

O diagnóstico definitivo do FEP se dá pela análise anatomopatológica do tumor. (STRECKER, et al., 2014)

O diagnóstico diferencial do FEP inclui, além das diversas patologias cardíacas e sistêmicas, trombos, vegetações, outros tumores (principalmente o mixoma) e a excrescência de Lambl. (PRIFTI, et al., 2015).

Pelo alto risco de obstruções e embolização sistêmica, após o diagnóstico é recomendada a ressecção cirúrgica a fim de evitar tais complicações. A abordagem de tratamento para pacientes sintomáticos é a exérese cirúrgica, preservando ao máximo o tecido valvar e sua função. Para os assintomáticos, a cirurgia ainda não tem indicação bem definida. A mobilidade do tumor é o fator determinante na indicação do tratamento cirúrgico. Os pacientes assintomáticos que não forem submetidos ao tratamento cirúrgico deveram fazer uso de anticoagulante para prevenir eventos embólicos, embora a eficácia deste uso seja questionável. (OLIVEIRA et al., 2005)

O paciente descrito possuía um quadro assintomático e diagnóstico incidental do tumor. Pela situação clínica, principalmente pela idade avançada, a equipe médica decidiu por não submeter o paciente a exérese cirúrgica, não tendo assim o diagnóstico por análise anatomopatológica.

\section{CONCLUSÃO}

O FEP é uma condição rara e de diagnóstico frequentemente incidental em nosso meio. Embora o caso relatado seja de um paciente assintomático e sem doenças prévias, vale ressaltar que as complicações do FEP - obstruções e embolizações sistêmicas, com consequente AVC e IAM - podem ser fatais. Nesse contexto, é importante pesquisar eventos prévios de origem coronariana ou cerebrovasculares. 
Relatamos um caso de FEP diagnosticado por ETT, embora sem confirmação anatomopatológica, em paciente octagenário, assintomático e sem eventos prévios que pudessem sugerir o diagnóstico.

O tratamento cirúrgico é a opção de eleição para a maioria dos casos, embora não tenha sido realizada no caso em tese, por decisão conjunta da equipe médica.

\section{REFERÊNCIAS}

CORSO, Ricardo Barros et al. Ruptura espontânea de angiossarcoma atrial direito e tamponamento cardíaco. Arq. Bras. Cardiol., São Paulo, v. 81, n. 6, p. 611-613, 2003. http://dx.doi.org/10.1590/S0066-782X2003001400008

GOUVEIA, Ana Carolina Nunes. Tumores cardíacos primários: classificação, diagnóstico e tratamento. 2010. Dissertação de Mestrado. Disponível em: https://estudogeral.uc.pt/bitstream/10316/31376/1/Trabalho\%20final\%20do\%206\%C 2\%BA\%20ano_Tumores\%20Card\%C3\%ADacos\%20Prim\%C3\%A1rios-

Classifica\%C3\%A7\%C3\%A30\%2C\%20diagn\%C3\%B3stico\%20e\%20tratamento..pdf Acesso em: 03 nov. 2018.

GOWDA et al. Cardiac papillary fibroelastoma: a comprehensive analysis of 725 cases. American Heart Journal, v. 146, n. 3, p. 404-410, 2003. https://doi.org/10.1016/S0002-8703(03)00249-7

KARIGYO, Carlos Junior Toshiyuki; SILVA, Felipe Batalini Freitas. Tumores cardíacos: uma breve revisão da literatura. Revista do Médico Residente, v. 16, n. 1, 2014. Disponível em: http://crmpr.org.br/publicacoes/cientificas/index.php/revistado-medico-residente/article/viewFile/528/513 Acesso em: 29 out. 2018.

LIMA, Paulo Ruiz Lucio de; CROTTI, Pedro Luís Reis. Tumores cardíacos malignos. Rev. bras. cir. cardiovasc, v. 19, n. 1, p. 64-73, 2004. http://dx.doi.org/10.1590/S0102-76382004000100012

MARISCALCO, Giovanni et al. Papillary fibroelastoma: insight to a primary cardiac valve tumor. Journal of cardiac surgery, v. 25, n. 2, p. 198-205, 2010. doi:10.1111/j.1540-8191.2009.00993.x

OLIVEIRA, Suzelle F. de M. et al. Fibroelastoma papilífero: experiência de uma Instituição. Arquivos Brasileiros de Cardiologia, v. 85, n. 3, p. 205-207, 2005.

http://dx.doi.org/10.1590/S0066-782X2005001600010

PRIFTI, Edvin et al. Papillary fibroelastoma of the anterior leaflet of the mitral valve mimicking vegetation. Journal of surgical case reports, v. 2015, n. 7, 2015. doi: 10.1093/jscr/rjv091

REYNEN, Klaus. Frequency of primary tumors of the heart. The American Journal of Cardiology, v 77, n. 1, p. 107, 1996. doi:10.1016/s0002-9149(97)89149-7

STRECKER, Thomas et al. Incidental papillary fibroelastoma of the tricuspid valve. Journal of cardiothoracic surgery, v. 9, n. 1, p. 123, 2014. doi:10.1186/17498090-9-123 